\title{
An International Comparative Analysis Highlighting that Canada is Not Keeping Pace With Patient Access to Drugs for Rare Diseases
}

Leanne Marie Ward

University of Ottawa Department of Pediatrics

Alexandra Chambers ( $\square$ alexandra.chambers@novartis.com )

Novartis Pharmaceuticals Canada Inc https://orcid.org/0000-0003-1250-2369

Emine Mechichi

Novartis Pharmaceuticals Canada Inc

Durhane Wong-Rieger

Canadian Organisation for Rare Disorders

Craig Campbell

London Health Sciences Centre Children's Hospital

\section{Research Article}

Keywords: Rare disease, orphan drugs, reimbursement, regulatory approval, funding decisions, patient access

Posted Date: August 3rd, 2021

DOI: https://doi.org/10.21203/rs.3.rs-725738/v1

License: (c) (7) This work is licensed under a Creative Commons Attribution 4.0 International License. Read Full License 


\section{Abstract}

Background: The Canadian government has committed to developing a national strategy for high-cost drugs for rare diseases starting in 2022. Considering this announcement, we conducted a comparative analysis to examine patient access to therapies for rare disease in each of Canada's 10 provinces relative to Europe and the U.S., to understand how Canada measures up relative to other countries.

Methods: We analyzed all of the therapies with an orphan drug designation approved by the European Medicine Agency (EMA) from 1 January 2015 to 31 March 2020 as the reference for the comparative analysis. We then contrasted access to these drugs in Canada (Health Canada) and the U.S. (Food and Drug Administration, FDA). Our analysis focused on: 1) the number of therapies for rare diseases entering the Canadian market; 2) the percentage of these therapies that are publicly available to Canadians; and 3) the timeliness for patient access to these therapies in Canada.

Results: Our analysis identified 63 approved therapies with an orphan drug designation from the EMA. The FDA has approved 53/63 (84\%) of these drugs, while Health Canada has approved 41/63 (65\%). In Europe, Germany, Denmark, and the U.K. had the highest percentage of publicly reimbursed orphan drugs (84\%, 70\%, 68\%, respectively). In comparison, Ontario (20/63, 32\%), Quebec (16/63, 25\%), and Alberta (16/63, 25\%) had the highest percentage of drugs reimbursed among the Canadian provinces. The shortest median duration (in months) from EMA approval to jurisdictional decision on reimbursement was in Austria (3.2), followed by Germany (4.1), and Finland (6.0). In Canada, the shortest median duration (in months) from regulatory approval to reimbursement was in British Columbia (17.3), Quebec (19.6) and Manitoba (19.6), while the longest duration was in P.E.I (38.5), followed by Nova Scotia (25.9), and Newfoundland (25.1).

Conclusions: Our comparative analysis found that Canadians had less frequent and timely access to therapies for rare disease than their global counterparts, highlighting the need for a strategy for rare disease in Canada that optimizes the number of patients with access to rare disease therapies, as quickly as possible.

\section{Background}

When considered as a whole, the prevalence of rare disease is about 4-6\% worldwide, which equates to $260-450$ million persons living with a rare disease at any given point in time. (1) Several therapies have been developed to treat and manage some of these rare diseases. Traditionally when therapies are developed to treat a specific disease, large clinical trials are conducted to demonstrate the efficacy and safety of the therapy. The results of these trials then inform decisions by health care systems around the world. In Canada, health care is administered provincially, whereby each province is responsible for its own delivery of health care within the mandate of the Canada Health Act. (2) Each province is charged with optimizing the delivery of care to their constituents.

An inherent challenge with therapies for rare diseases is that it is logistically difficult to conduct large, robust clinical trials for several reasons, not the least of which is the small number of patients in any one region or country. When larger more methodologically traditional clinical trials cannot be conducted, health care systems have difficulty under the current evaluation paradigms in assessing the value of the therapy for rare disease. Many countries have developed strategies to overcome the obstacles regarding access for rare diseases. (3) These strategies include creating unique mechanisms for the review of rare disease therapies, creating special budgets for these interventions, and developing preferential 'scoring' of therapies for rare diseases compared to treatments for other conditions. (4) While this challenge to generate supporting evidence to integrate novel therapies into rare disease management strategies has been acknowledged in Canada (5), currently there are no there are no mechanisms to support the drug access process that specifically target therapies for rare diseases in this country. Recently the Canadian federal government committed \$1 billion to launch a National Strategy for High-Cost Drugs for Rare Diseases starting in 2022. (6) As of May 2021, the federal consultations on the strategy have indicated a focus on therapies associated with high cost, high unmet need, and high uncertainty; however, the details on the governance, structure, and funding allocation remain unclear. Nonetheless, patients with rare diseases and the clinicians who treat them welcome the spirit behind the initiative.

Canada's process for the public reimbursement of novel drugs is multi-step and complex (Figure 1). All drugs entering the Canadian market first require regulatory approval by Health Canada; however, there are several steps after a drug receives Health Canada approval that are required before a drug is accessible to patients on a public formulary. Each step can take several months to complete, creating concern among clinicians and patients about ensuring timely access to therapies. After regulatory approval by Health Canada, in order for a drug to be considered for public listing on a provincial formulary, it must proceed through the Health Technology Assessment (HTA) pathway. Canada has 2 HTA agencies (Canadian Agency for Drugs and Technologies in Health [CADTH] and Institut National d'Excellence en Santé et Services Sociaux [INESSS, Quebec only]) that review the value of the drug compared to existing therapies and make recommendations to the public drug plans on whether the drug should be reimbursed. If a drug receives a positive recommendation from an HTA agency, the next step in the process is to negotiate a price with the pan Canadian Pharmaceutical Alliance (pCPA) that can be used across Canada's public drug plans. The final step in the process is for each province to make its own funding decision for the drug under review based on the province's budget and other priorities. Access for public and private drug coverage varies in Canada and access varies significantly across private plans depending on the specific plan sponsor. Since most Canadian patients with rare diseases rely on public drug reimbursement (for example, as a catasatrophic cost outside of the private plan), it is appropriate to focus on the public drug plans for the purposes of comparison with European public reimbursement.

In light of the commitment to a national strategy for high-cost drugs for rare diseases that endeavours to overcome these barriers in Canada, we undertook a novel analysis to understand how Canada compares to other countries on access to therapies for rare diseases. Our analysis focused on three key variables: 1) the number of therapies for rare disease entering the Canadian market compared to the European Medicines Agency (EMA and the U.S. Food and Drug Administration (FDA); 2) the percentage of these therapies that are publicly available to Canadians; and 3) the timeliness for access to these therapies.

\section{Methods}


We generated a list of drugs with an orphan drug designation approved by the EMA from 1 January 2015 to 31 March 2020 . We chose this time period because we did not want any impact of the COVID-19 pandemic to unduly influence the observations, and this timing aligns with the U.K.'s gradual transition from EMA to using their own agency, Medicines and Healthcare Products Regulatory Agency (MHRA), for regulatory approvals. (7) As a result, the approval decisions made by the EMA also applied to the U.K. in this time period.

For context, we also compared the list of drugs with an orphan designation in the EMA with the FDA. Both the EMA and FDA have criteria for orphan drug designations. These regulatory agencies use slightly different definitions to establish orphan drug status, notably the FDA's criteria are broader than the EMA's (Table 1). For this comparative analysis, we used the EMA's criteria when creating the final list of drugs to use for the analysis because of the similarity in the health care systems in Europe compared to Canada, and both Health Canada and the Canadian Institute of Health Research (CIHR) reference the EMA orphan drug definition in their documentation $(8,9)$ For these reasons, we considered the EMA definition was more appropriate for the Canadian context than the FDA's. Using publicly available data from the EMA, FDA, and Health Canada, we established the status and dates of regulatory approval for the list of drugs that we generated using the EMA's orphan drug designation. (Table 2)

\section{Determining the percentage of the rare disease therapies that are publicly available in Canada}

For the drugs with Health Canada approval, we went on to determine the status and date of the HTA recommendation from both of Canada's HTA agencies: CADTH and INESSS. If a drug received a positive recommendation from either HTA agency, we identified the date and status of the pan Canadian Pharmaceutical Alliance ( $P C P A)$ negotiations for a confidential drug price applicable to the Canadian public drug plans. And finally, if there was a pricing agreement reached on a $P C P A$ negotiation for a drug, we searched the public websites of each of the provincial formularies to determine which provinces publicly reimbursed the drug. All data regarding HTA recommendations, pCPA negotiations, and provincial funding decisions were derived from the public data sources listed in Table 3.

Our analysis focused on therapies that are publicly reimbursed by the provinces. The primary objective of the Canada Health Act is to protect, promote and restore the physical and mental well-being of residents of Canada and to facilitate reasonable access to health services without financial or other barriers. (2) We recognize that many Canadians have access to private insurance that may provide access to therapies for rare disease; however, private coverage was beyond the scope of this analysis.

\section{Benchmarking the timeliness of patient access to the therapies for rare diseases}

Upon extraction of data from the various stages of the drug access process, we measured the timelines between each step of the drug access process and compared patient access to these therapies across the ten Canadian provinces. We excluded Canada's three territories from the analysis because of their substantial reliance on federal drug program funding and their relatively small share of the Canadian population (the three territories combined account for less than $1 \%$ of Canada's total population). (27)

\section{Comparing patient access in Canada to Europe}

Market access was assumed in each Canadian province when the therapy was publicly listed on the provincial ministry of health formulary website. Generally, we determined that a drug for a rare disease was reimbursed in the European countries if it had a recommended (or partially recommended) funding status, it was listed on a national reimbursement list, or it was noted to have case-by-case reimbursement. We determined a drug was not reimbursed if there was a negative recommendation, no decision, or a decision pending. Every jurisdiction (province, country) has a different health system for providing market access. In order to conduct the comparative analysis several assumptions were made regarding access to therapy in each jurisdiction. Table 4 outlines these assumptions and exceptions.

We compared the timeline from regulatory approval until reimbursement in Europe and Canada. For the European countries we measured the median difference from the date of EMA approval to the date of reimbursement decision in each country. For the Canadian provinces we measured the median difference of the date of the Health Canada approval to the date of the reimbursement decision in each of the provinces.

\section{Results}

\section{Number of therapies for rare diseases entering the Canadian market}

Between 1 January 2015 and 31 March 2020, the EMA approved 63 drugs with an orphan drug designation (Table 5). An additional 3 drugs were granted orphan designation by the EMA, but ultimately did not receive EMA approval. The FDA has approved 53 (84\%) of these drugs for use in the United States. Health Canada has approved 41 (65\%) of these drugs for use in Canada. Notably, the FDA approved 356 drugs with orphan designation in this same time period which can likely be attributed to the fact that the FDA criteria for orphan drug designation are broader than the EMA criteria (Table 1).

After regulatory approval by Health Canada, a drug is submitted for an HTA review to establish if there is value for the drug compared to the existing therapies for the same indication. We found that of the 41 drugs with Health Canada approval, 36/41 (88\%) had undergone an HTA review by CADTH, and 33/41 (80\%) drugs had undergone an HTA review by INESSS (Quebec only). Of CADTH's 36 HTA reviews, they issued positive recommendations for $30 / 36$ ( $83 \%$ ) and negative recommendations for $6 / 36$ (17\%), with one review still ongoing as of 21 May 2021. Of INESSS's 33 HTA reviews, they issued positive recommendations for $20 / 33(61 \%)$ and negative recommendations for 13/33 (39\%) reviews. In eight of 33 reviews, INESSS and CADTH made different 
recommendations whereby INESSS issued negative recommendations for all of the eight drug reviews and CADTH's issued positive recommendations for the same therapies. (Figure 2).

When we reviewed the status of the drugs at the price negotiation step with the PCPA, we identified that 24 drugs had an agreement with pCPA on a confidential price for the drug. In addition, there were seven ongoing negotiations as of 21 May 2021. There was one instance where despite a positive CADTH recommendation, a pricing agreement was not reached with pCPA (Eliglustat (Cerdelga) for Gaucher Disease). There was an active negotiation with pCPA where both CADTH and INESSS issued a negative recommendation, yet pCPA was still pursuing a pricing negotiation (Ivacaftor \& Lumacaftor (Orkambi) for cystic fibrosis).

\section{Proportion of rare disease therapies publicly available to Canadians}

Since each province makes its own public funding decisions, there was variation observed across the country in terms of access to therapies for rare diseases. When we took the list of 63 EMA-approved orphan designated drugs we found that less than one-third of these drugs were publicly funded. Ontario had the most public patient access of the provinces with 20/63 (32\%) of the drugs publicly reimbursed, followed by Quebec (16/63, 25\%), and Alberta (16/63, 25\%). On the other hand, Newfoundland $(7 / 63,11 \%)$ and Prince Edward Island (PEI) $(2 / 63,3 \%)$ had the least publicly funded access to these therapies. (Figure 3 )

To provide additional context, our analysis compared the percentage of therapies for rare disease approved by the EMA that were available to patients in 27 European countries to the data from the Canadian provinces (Figure 4). When we looked at the analysis, we found that Germany had the highest access to the therapies for rare diseases approved by the EMA (84\%), followed by Denmark (70\%) and the U.K. (68\%).

For simplicity, we also compared the Canadian provinces to the EU5 (Germany, Italy, Spain, France, U.K.). The EU5 represents that five largest European drug markets. (28) As mentioned previously, this drug access period observed for this analysis was primarily prior to the U.K.'s departure from the European Union. Figure 5 illustrates that access was lower in all provinces compared to the EU5.

\section{Timeliness of the Canadian patient access pathway}

Of the 41 drugs with Health Canada approval that fell within the 63 drugs with EMA orphan drug designation, we found that $7 / 41$ (17\%) of these drugs were approved by Health Canada before the EMA. When we compared the difference in regulatory approval timelines between EMA, FDA, and Health Canada we observed closer timelines between the EMA and FDA, compared to Health Canada and EMA or Health Canada and FDA. (Figure 6) The median lag time for EMA approval after FDA approval was 6.1 months compared to the median lag time of 11.0 months for Health Canada after FDA approval. There was substantial of discrepancy in the mean and median lag time for Health Canada after EMA approval highlighting a wide variation in time lag (range -4.1 to 41.2 months) between the 2 regulators.

When we analyzed the time lag for each step in the Canadian drug access pathway from Health Canada approval to a pCPA (price negotiation) agreement, we observed that each step takes approximately 5 to 10 months (Figure 7). The median time from Health Canada approval to pCPA agreement was 14.8 months. Overall, we observed the median time from the CADTH HTA recommendation to completion with pCPA was 9.9 months, and 8.2 months from the INESSS recommendation to a PCPA agreement.

When we looked at the timeline from Health Canada approval to provincial reimbursement, we found disparity in the timeline to make a funding decision. This is notable because all of the provinces would have followed the same timeline to the point of a pCPA agreement (median 14.8 months). The last step in the process whereby each province makes a funding decision is the most variable(Figure 8 ). British Columbia made the fastest decisions, with a median of 1.2 months, regarding provincial funding once a pCPA agreement had been reached. PEI had the longest median time lag between the pCPA agreement and a provincial funding decision at $\mathbf{2 3 . 7}$ months. It such be noted, however, that there were only two drugs identified that were publicly reimbursed in PEl, so the sample was very small.

\section{Timeliness of access to therapies for rare disease compared to Europe}

We compared the timeline from regulatory approval until reimbursement across 23 European countries to the Canadian provinces' timeline for orphan designation drugs (Figure 9). Based on our analysis, Austria had the shortest median timeline from regulatory approval to reimbursement decision (3.2 months), and Poland had the longest median timeline (43.6 months). The Canadian provinces had a median timeline of 1 to 2 years between Health Canada approval and public reimbursement, with the exception of Newfoundland and PEl which both had median timelines beyond 2 years. It is important to use caution interpreting these latter two results since there were only two drugs reimbursed by PEl, and seven by Newfoundland, to inform the timeline calculations.

\section{Discussion}

We undertook this analysis to compare access to therapies for rare diseases across Canada and to Europe because Canada is in the process of developing a National Strategy for High-Cost Drugs for Rare Diseases. (6) We thought that this timely and novel analysis was important to understand how Canada fared by province and in comparison, to other countries. Our primary analysis focused on drugs for rare diseases approved by the EMA with an orphan drug designation. We chose the EMA because of the similarity of the Canadian health system to the health systems of many European countries and the paradigm of public payer reimbursement being more congruent. However, we also decided to include which of the EMA approved drugs that had also been approved by the U.S. FDA. We did this because of the size, prominence, and close proximity of the U.S to Canada; and the interdigitation of the rare disease patient community. We recognize that there are many differences between the health systems in Canada and the U.S., and that FDA approval alone does not equate to drug access for all Americans. 
Our analysis identified two key findings: 1) we found that fewer therapies for rare disease were submitted to Health Canada than to the EMA or FDA for approval, and 2) that among those submitted to Health Canada, less than half were reimbursed on a public formulary in any province. These findings raise a number of questions about the reasons for such discrepancy. Why are fewer therapies for rare diseases being submitted for regulatory approval in Canada compared with the EU and the U.S., and on a later timeline? Why are comparatively fewer therapies ultimately approved for public reimbursement following health authority approval in Canada, relative to the EU? Are the mechanisms for measuring therapy value in Canada not congruent with those mechanisms in the EU?

\section{Disparity between Health Canada and EMA Approvals}

This comparative analysis has identified that fewer than two-thirds of the therapies approved by the EMA are even being submitted to Health Canada for regulatory approval. And while it is easy to blame the fact that Canada does not have a rare disease strategy in place, it is important to truly understand the component parts leading to this discrepancy. Could this be due to the small population of Canada relative to Europe, and the resources required for a pharmaceutical company to bring their drug to Canada is not considered a good return on investment? Another possibility is the link between clinical trial activity in a country/region and the decision to bring to regulatory review. This was this was beyond the scope of our study but would be an important factor to examine in future work. Without further research, we cannot fully understand why there is the disparity in submissions to Health Canada compared with Europe. In 2019, Project Orbis was launched by the FDA to partner with other regulatory agencies, including Health Canada, with the aim of providing "patients faster access to promising cancer treatments across the globe". (29) While Project Orbis is strictly focused on cancer therapies at this time, if proven successful, this program or similar models have the potential to be expanded to other therapeutic areas. Uncovering the factors leading to the rationale for the lower number of submissions made to Health Canada compared with the EMA, will provide an opportunity to collectively strategize with clinicians, patient groups, federal and provincial governments, and the pharmaceutical industry on how to increase the number of drugs entering the Canadian market for approval.

\section{Low Rate of Public Reimbursement of Drugs with Health Canada Approval}

Beyond the issue of the number of therapies entering the Canadian market, there is the concerns of the low number of therapies that successfully navigate the Canadian drug access pathway from Health Canada approval to public reimbursement on a provincial formulary. Our analysis found that half of the therapies submitted to Health Canada for approval proceeded to the point of a successful price negotiation with the pCPA, and then an even smaller proportion were found to be publicly funded in each province. This imbalance in access across provinces raises genuine equity concerns that warrant further exploration. An important subsequent research question would be to further understand why only half of the drugs progressed to this step: 1) was it due to uncertainty in the clinical evidence?; 2) was it because there was already an effective standard of care and this new therapy was not assessed as adding clinical value?; or 3 ) was it because the cost of the therapy was perceived to be prohibitive and avaricious? Are there patient organization advocacy or real world evidence solutions that work on the positive side to sustain momentum through the approval process? If we can understand the positive and negative pressure points in the pathway, there is an opportunity to develop solutions or mechanisms to increase patient access to effective therapies.

The due diligence and rigour to ensure that a therapy is effective, safe, and affordable within the health system are critical for the sustainability of the health system. At the same time, these standards must be balanced against the pressing need to provide adequate treatments for those with debilitating or even lifethreatening illnesses, in order to provide access to therapy that could improve or save lives. This balance, which further depends on a sustainable, fair access funding program, emphasizes the importance of a rare disease strategy that provides optimal therapy for patients so that, like those with common disorders such as cardiovascular disease, chronic respiratory diseases, and diabetes, have an equal opportunity to live their best lives.

For many years, there have been articles written about the need for real world evidence $(30,31)$ and outcomes-based agreements $(32,33)$ to address issues of uncertainty of the clinical value of therapies. In 2014, the provincial Ministers of Health in Canada developed an Expensive Drug for Rare Disease working group to pilot a proposal for supplemental processes for complex/specialized drugs that builds upon existing review processes with health partners. (34,35) The work of the Expensive Drugs for Rare Disease working group has yet to be delivered publicly. Understandably there is a concern in overwhelming an already overburdened drug access pathway by introducing new processes that require more time and resources to implement, however, there are opportunities to gain efficiency such as providing conditional access to drugs while gathering real world evidence to reduce the uncertainty in the HTA review . The rare disease community is an ideal setting to explore some of these mechanisms because of the small patient populations and, typically, the unmet need for effective therapies for patients. The Canadian Organization for Rare Disorders (CORD) has been actively engaged in supporting a robust and comprehensive strategy to support patients with rare diseases for several years, including most recently hosting consultations with a wide array of stakeholders to support the National Strategy for High-Cost Drugs for Rare Diseases in Canada. (36)

\section{Limitations}

We acknowledge that our comparative analysis has several limitations. This analysis was a one-way assessment looking at drugs that were given an orphan drug designation by the EMA and following those drugs through the reimbursement process in Canada. This analysis did not do the reverse and look for Health Canada-approved drugs that would have met the criteria for an EMA orphan designation but were not approved by the EMA. Also, we relied only on publicly available sources of information, and some provinces may provide access to some therapies on a case-by-case basis that is not publicly available. Therefore, any therapies that are provided on a case-by-case basis were not captured in this analysis. Nevertheless, since Canada's health care system is predicated upon equal access to all, a reasonable first step was to examine the progress of access to rare disease therapies in the public domain.

\section{Conclusions}


Rare diseases impact many people globally, and we are likely to see a continued effort by the research community to develop more new therapies for the multitude of conditions that fall into the broad catchment of rare disease. Our comparative analysis has unveiled that Canadians have less access to therapies for rare disease than those in other countries. In order for Canada to improve its record, we need a coordinated national strategy that will enable patients with rare disease to equitably and sustainably receive timely access to the most effective therapies. We are encouraged that the Canadian federal government recognizes the need to have a strategy for high cost drugs for rare diseases, and hope that this leads to a comprehensive rare disease strategy. (6) In support of such a strategy, we recommend further analysis to understand the root causes of 1) the disparity in drugs for rare disease entering the Canadian market compared to the EU, and 2) the considerable proportion of Health Canada-approved therapies that are not publicly reimbursed. Once we can understand these root causes and use them to inform the National Strategy for High-Cost Drugs for Rare Diseases in Canada such that it results in improved access for Canadians who would benefit most from emerging therapies.

\section{List Of Abbreviations}

- CADTH Canadian Agency for Drugs and Technologies in Health

- EMA European Medicines Agency

- EU European Union

- FDA Food and Drug Administration

- HTA Health Technology Assessment

- INESSS Institut national d'excellence en santé et services sociaux

- pCPA pan Canadian Pharmaceutical Alliance

\section{Declarations}

Ethics approval and consent to participate

Not applicable

Consent for publication

Not applicable

Availability of data and materials

All data were extracted from public sources

Funding

Novartis Pharmaceuticals funded the analysis

Disclosures:

LMW has participated in clinical trials with, and/or been a consultant to, PTC, ReveraGen, Catabasis, Amgen, Novartis, Alexion, Ascendis, Ipsen, and Ultragenyx, with funds to LMW's institution.

AC and EM are employed by Novartis Pharmaceuticals

Authors' contributions

AC contributed to the study design, coding and analysis of study data, and manuscript writing. EM participated in data collection, coding and analysis of study data, and manuscript writing. LW, DWR, and CC contributed to the study concept and review of the manuscript. All authors read and approved the final manuscript

Acknowledgements

LMW is supported by a Tier 1 Clinical Research Chair at the University of Ottawa

\section{References}

1. Nguengang Wakap S, Lambert DM, Olry A, Rodwell C, Gueydan C, Lanneau V, et al. Estimating cumulative point prevalence of rare diseases: analysis of the Orphanet database. Eur J Hum Genet. 2020;28(2):165-73.

2. Health Canada. Canada Health Act [Internet]. Canada.ca. 2004 [cited 2021 May 29]. Available from: https://www.canada.ca/en/healthcanada/services/health-care-system/canada-health-care-system-medicare/canada-health-act.html

3. Impact HTA [Internet]. Impact-hta.eu. [cited 2021 May 29]. Available from: https://www.impact-hta.eu/country-vignettes

Page 6/16 
4. Nicod E, Whittal A, Drummond M, Facey K. Are supplemental appraisal/reimbursement processes needed for rare disease treatments? An international comparison of country approaches. Orphanet J Rare Dis. 2020;15(1):189.

5. Richter T, Janoudi G, Amegatse W, Nester-Parr S. Characteristics of drugs for ultra-rare diseases versus drugs for other rare diseases in HTA submissions made to the CADTH CDR. Orphanet J Rare Dis [Internet]. 2018;13(1). Available from: http://dx.doi.org/10.1186/s13023-018-0762-1

6. Health Canada. National strategy for high cost drugs for rare diseases online engagement - Canada.Ca [Internet]. Canada.ca. 2021 [cited 2021 May 29 ]. Available from: https://www.canada.ca/en/health-canada/programs/consultation-national-strategy-high-cost-drugs-rare-diseases-online-engagement.html

7. Europa.eu. [cited 2021 May 29]. Available from: https://www.ema.europa.eu/en/about-us/brexit-united-kingdoms-withdrawal-european-union

8. Lee DK, Wong B. An orphan drug framework (ODF) for Canada. J Popul Ther Clin Pharmacol. 2014;21(1):e42-6.

9. Government of Canada, Canadian Institutes of Health Research, Institutes, Institute of Genetics. Supporting cutting-edge rare disease research [Internet]. Cihr-irsc.gc.ca. 2016 [cited 2021 May 29]. Available from: https://cihr-irsc.gc.ca/e/49695.html

10. Office of the Commissioner. Designating an orphan drug or biologic [Internet]. Fda.gov. 2021 [cited 2021 May 29]. Available

from: https://www.fda.gov/industry/developing-products-rare-diseases-conditions/designating-orphan-product-drugs-and-biological-products

11. Health Canada. Notice of Compliance (NOC) database [Internet]. Canada.ca. 2005 [cited 2021 May 29]. Available from: https://www.canada.ca/en/healthcanada/services/drugs-health-products/drug-products/notice-compliance/database.html

12. Search orphan drug designations and approvals. [cited 2021 May 29]; Available

from: https://www.accessdata.fda.gov/scripts/opdlisting/oopd/index.cfm

13. Reimbursement Review Reports [Internet]. Cadth.ca. 2015 [cited 2021 May 29]. Available from: https://www.cadth.ca/reimbursement-review-reports

14. INESSS [Internet]. Inesss.qc.ca. [cited 2021 May 29]. Available from: https://www.inesss.qc.ca/thematiques/medicaments/medicaments-evaluation-auxfins-dinscription.html

15. Brand Name Drug Negotiations Status [Internet]. Pcpacanada.ca. [cited 2021 May 29]. Available from: https://www.pcpacanada.ca/negotiations 16. Ministry of Health. Drug Coverage [Internet]. Gov.bc.ca. [cited 2021 May 29]. Available from: https://www2.gov.bc.ca/gov/content/health/health-drugcoverage/pharmacare-for-bc-residents/what-we-cover/drug-coverage

17. Alberta health - drug benefit list [Internet]. Bluecross.ca. [cited 2021 May 29]. Available from: https://idbl.ab.bluecross.ca/idbl/load.do

18. Home Page - Online Formulary 2021 [Internet]. Ehealthsask.ca. [cited 2021 May 29]. Available

from: http://formulary.drugplan.ehealthsask.ca/SearchFormulary

19. Author's Name. Pharmacare Program [Internet]. Gov.mb.ca. [cited 2021 May 29]. Available from: https://web22.gov.mb.ca/eFormulary/

20. Check medication coverage [Internet]. Ontario.ca. [cited 2021 May 29]. Available from: https://www.ontario.ca/page/check-medication-coverage/

21. Government of Ontario, Ministry of Health, Care L-T. Exceptional Access Program - Formulary - health care professionals - MOHLTC. 2009 [cited 2021 May 29]; Available from: https://www.health.gov.on.ca/en/pro/programs/drugs/odbf/odbf_except_access.aspx

22. Médicaments [Internet]. Gouv.qc.ca. [cited 2021 May 29]. Available

from: https://www.ramq.gouv.qc.ca/fr/professionnels/pharmaciens/medicaments/Pages/liste-medicaments.aspx

23. Government of New Brunswick, Canada. New Brunswick Drug Plans Formulary [Internet]. Gnb.ca. 2014 [cited 2021 May 29]. Available

from: https://www2.gnb.ca/content/gnb/en/departments/health/MedicarePrescriptionDrugPlan/NBDrugPlan/ForHealthCareProfessionals/NewBrunswickDru

24. Novascotia.ca. [cited 2021 May 29]. Available from: https://novascotia.ca/dhw/pharmacare/formulary

25. Health and Community Services [Internet]. Gov.nl.ca. [cited 2021 May 29]. Available

from: https://www.health.gov.nl.ca/health/prescription/newformulary.asp

26. PEI Pharmacare Formulary [Internet]. Princeedwardisland.ca. 2019 [cited 2021 May 29]. Available

from: https://www.princeedwardisland.ca/en/information/health-pei/pei-pharmacare-formulary

27. Brandt J, Shearer B, Morgan SG. Prescription drug coverage in Canada: a review of the economic, policy and political considerations for universal pharmacare. J Pharm Policy Pract. 2018;11(1):28.

28. Espin J, Schlander M, Godman B, Anderson P, Mestre-Ferrandiz J, Borget I, et al. Projecting pharmaceutical expenditure in EU5 to 2021: Adjusting for the impact of discounts and rebates. Appl Health Econ Health Policy. 2018;16(6):803-17. 
29. Health Canada. Project Orbis [Internet]. Canada.ca. 2021 [cited 2021 May 29]. Available from: https://www.canada.ca/en/health-canada/services/drugshealth-products/international-activities/project-orbis.html

30. Wang SV, Pinheiro S, Hua W, Arlett P, Uyama Y, Berlin JA, et al. STaRT-RWE: structured template for planning and reporting on the implementation of real world evidence studies. BMJ. 2021;372:m4856.

31. Khosla S, White R, Medina J, Ouwens M, Emmas C, Koder T, et al. Real world evidence (RWE) - a disruptive innovation or the quiet evolution of medical evidence generation? F1000Res. 2018;7:111.

32. Toumi M, Jarosławski S, Sawada T, Kornfeld Å. The use of surrogate and patient-relevant endpoints in outcomes-based Market Access Agreements: Current debate: Current debate. Appl Health Econ Health Policy. 2017;15(1):5-11.

33. Dabbous M, Chachoua L, Caban A, Toumi M. Managed entry agreements: Policy analysis from the European perspective. Value Health. 2020;23(4):42533.

34. Cadth.ca. [cited 2021 May 29]. Available from: https://cadth.ca/sites/default/files/symp-2019/presentations/april15-2019/A1-presentation-elun.pdf

35. Raredisorders.ca. [cited 2021 May 29]. Available from: http://www.raredisorders.ca/content/uploads/EDRD-supplemental-process-

background_240ct2018_Final.pdf

36. Time to jump start rare disease drug strategy [Internet]. Raredisorders.ca. [cited 2021 Jul 16]. Available from: http://www.raredisorders.ca/time-to-jumpstart-rare-disease-drug-strategy/

\section{Tables}

Table 1. Comparison of FDA and EMA criteria for orphan drug designation

$\begin{array}{ll}\text { FDA Criteria (10) } & \text { EMA Criteria (7) }\end{array}$

- Drugs (includes biologics) for the prevention, diagnosis, or treatment of diseases or conditions affecting fewer than 200,000 persons in the US OR

- Drugs that will not be profitable within 7 years following approval by the FDA

- The drug must be intended for a disease that is lifethreatening or chronically debilitating;

- The prevalence of the condition must not be more than 5 in 10,000; AND

- The medicine must be of significant benefit to those affected by the condition.

Abbreviations: EMA, European Medicines Agency; FDA, Food and Drug Administration

Table 2. Sources of data used to determine regulatory approval (links accessed May 13, 2021)

\begin{tabular}{|ll|}
\hline Agency & Data Source \\
\hline European Medicine Agency (EMA) & $\begin{array}{l}\text { EMA Medicines Database (7): } \\
\text { https://www.ema.europa.eu/en/medicines }\end{array}$ \\
\hline Health Canada & Notice of Compliance (NOC) Database (11): \\
& $\begin{array}{l}\text { https://www.canada.ca/en/health-canada/services/drugs-health-products/drug-products/notice- } \\
\text { compliance/database.html }\end{array}$ \\
$\begin{array}{l}\text { U.S. Food and Drug Administration } \\
\text { (FDA) }\end{array}$ & FDA Orphan Drug Designation Database (12): \\
\hline
\end{tabular}

Due to technical limitations, table 3 is only available as a download in the Supplemental Files section.

Table 4. Assumptions used to determine availability of the therapy for rare disease 


\begin{tabular}{|c|c|c|}
\hline Country/Province & Reimbursement Status & Timeline of reimbursed access \\
\hline Austria & $\begin{array}{l}\text { Medicine is available if it is included in the reimbursement system on } \\
\text { Austrian pharmacies list }\end{array}$ & Date of appearance in Austrian pharmacies list \\
\hline Belgium & $\begin{array}{l}\text { Medicine is available if it is on the reimbursement list, or funded through } \\
\text { the hospital }\end{array}$ & Date of appearance in INAMI \\
\hline Bulgaria & Medicine is available if it is on the reimbursement list & Date of publication in Positive drug list - NCPR register \\
\hline $\begin{array}{l}\text { Canada (all } \\
\text { provinces) }\end{array}$ & Medicine is available if it is on the reimbursement list & Date listed on website \\
\hline Croatia & Medicine is available if it is on the reimbursement list & $\begin{array}{l}\text { Date of publication in CHIF, Croatian Health Insurance } \\
\text { Fund }\end{array}$ \\
\hline Cyprus & Medicine is available if it is on the official $\mathrm{MOH}$ price list & Date of inclusion in official price list \\
\hline Czech Republic & Medicine is available if it is on the reimbursement list & $\begin{array}{l}\text { Date of inclusion in official reimbursement list } \\
\text { published by SUKL }\end{array}$ \\
\hline Denmark & $\begin{array}{l}\text { Medicine is available if has received public reimbursement by Danish } \\
\text { Medical agency, No information (not available) for case by case individual } \\
\text { reimbursements }\end{array}$ & Date of inclusion in official reimbursement list \\
\hline Finland & $\begin{array}{l}\text { Medicine is available if it is on the Pharmaceuticals Pricing Board } \\
\text { publication list. }\end{array}$ & $\begin{array}{l}\text { Date of inclusion in PPB pricing and reimbursement } \\
\text { list }\end{array}$ \\
\hline France & $\begin{array}{l}\text { Medicine is available if it is on the reimbursement list, or funded through } \\
\text { the hospital }\end{array}$ & $\begin{array}{l}\text { HTA body decision date considered form Drug Context } \\
\text { Matters Market Access Platform and France MOH } \\
\text { website. }\end{array}$ \\
\hline Germany & $\begin{array}{l}\text { Medicine is available if it received positive recommendation received from } \\
\text { GBA, IQVIG }\end{array}$ & $\begin{array}{l}\text { HTA body decision date considered form Drug Context } \\
\text { Matters Market Access Platform. }\end{array}$ \\
\hline Greece & Medicine is available if it is on the reimbursement list & $\begin{array}{l}\text { Date of inclusion in official price publication by Greece } \\
\mathrm{MOH}\end{array}$ \\
\hline Hungary & Medicine is available if it is on the reimbursement list & Date of inclusion in official NHIF publication \\
\hline Ireland & Medicine is available if it is on the reimbursement list & Date of inclusion in Ireland monthly price update \\
\hline Italy & $\begin{array}{l}\text { Medicine is available if it is on the reimbursement list, or funded through } \\
\text { the hospital }\end{array}$ & $\begin{array}{l}\text { HTA body decision date considered form DRG Context } \\
\text { Matters Market Access Platform and AIFA website. }\end{array}$ \\
\hline Luxembourg & $\begin{array}{l}\text { Medicine is available if available in Belgium - automatic reimbursement } \\
\text { following Belgium and official price list }\end{array}$ & $\begin{array}{l}\text { Date of inclusion in official price publication, PriCentric } \\
\text { Launch dates }\end{array}$ \\
\hline Netherlands & Medicine is available if it is on the reimbursement list & $\begin{array}{l}\text { Date of inclusion in official reimbursement list of } \\
\text { Netherlands } \mathrm{MOH}\end{array}$ \\
\hline Norway & Medicine is available if it is on the reimbursement list & $\begin{array}{l}\text { Date of inclusion in official reimbursement list of } \\
\text { Norway } \mathrm{MOH}\end{array}$ \\
\hline Poland & Medicine is available if it is on the reimbursement list & $\begin{array}{l}\text { Date of inclusion in official reimbursement list of } \\
\text { Poland } \mathrm{MOH}\end{array}$ \\
\hline Romania & $\begin{array}{l}\text { Medicine is available if the PriCentric database shows as reimbursed; } \\
\text { Latest information in official Romania } \mathrm{MOH} \text { publication is missing }\end{array}$ & Date of Launch in the PriCentric Database \\
\hline Slovakia & Medicine is available if it is on the official price list & $\begin{array}{l}\text { Date of inclusion in Lists of officially determined prices } \\
\text { - Slovakia MOH, PriCentric launch date }\end{array}$ \\
\hline Slovenia & Medicine is available if it is on the Central Medicines Database & Central Medicines Database, PriCentric Launch date \\
\hline Spain & $\begin{array}{l}\text { Medicine is available if it received positive recommendation received from } \\
\text { Spain } \mathrm{MOH} \text {. }\end{array}$ & $\begin{array}{l}\text { HTA body decision date considered form Drug Context } \\
\text { Matters Market Access Platform. }\end{array}$ \\
\hline Sweden & Medicine is available if it is listed on TLV drug database. & $\begin{array}{l}\text { Timelines according to TLV drug database "Prices } \\
\text { Valid from" date }\end{array}$ \\
\hline Switzerland & $\begin{array}{l}\text { Medicine is available if it is on the official reimbursement list of Swiss } \\
\mathrm{MOH} \text {. }\end{array}$ & Date of inclusion in PriCentric database. \\
\hline U.K. & $\begin{array}{l}\text { Medicine is available if it received positive recommendation received from } \\
\text { NICE }\end{array}$ & $\begin{array}{l}\text { HTA body decision date considered form DRG Context } \\
\text { Matters Market Access Platform, PriCentric } \\
\text { Reimbursement date }\end{array}$ \\
\hline
\end{tabular}

Table 5. Drugs with EMA orphan designation approved from Jan 2015 to Mar 2021 


\begin{tabular}{|c|c|c|}
\hline Generic Name & Brand Name & Indication \\
\hline Asfotase Alfa & Strensiq & Hypophosphatasia \\
\hline Avelumab & Bavencio & Merkel Cell Carcinoma \\
\hline Axicabtagene Ciloleucel & Yescarta & Primary Mediastinal Large B-Cell Lymphoma, Large B-Cell Lymphoma \\
\hline Budesonide & Jorveza & Eosinophilic Esophagitis \\
\hline Burosumab & Crysvita & Hypophosphatemia (X-Linked) \\
\hline Cannabidiol & Epidyolex & Seizures (Lennox-Gastaut Syndrome), Dravet Syndrome \\
\hline Caplacizumab & Cablivi & Thrombotic Thrombocytopenic Purpura \\
\hline cenegermin-bkbj & Oxervate & Neurotrophic keratitis \\
\hline Cerliponase Alfa & Brineura & Neuronal Ceroid Lipofuscinosis Type 2 \\
\hline Chlormethine & Ledaga & Cutaneous T-Cell Lymphoma \\
\hline Coagulation Factor Ix & Idelvion & Haemophilia B \\
\hline Coagulation Factor Ix & Alprolix & Haemophilia B \\
\hline Coagulation Factor $\mathrm{X}$ & Coagadex & Factor X Deficiency \\
\hline Cytarabine \& Daunorubicin & Vyxeos & AML \\
\hline Daratumumab & Darzalex & Multiple Myeloma \\
\hline Darvadstrocel & Alofisel & Crohn'S Disease (Fistulising) \\
\hline Dinutuximab Beta & Unituxin & Neuroblastoma \\
\hline Eliglustat & Cerdelga & Gaucher Disease \\
\hline Gallium (68Ga) Edotreotide & Somakit Toc & Diagnostic Use (Gastro-Entero-Pancreatic Neuroendocrine Tumours) \\
\hline Gemtuzumab Ozogamicin & Mylotarg & AML \\
\hline Gilteritinib & Xospata & Aml (Flt3+) \\
\hline Givosiran & Givlaari & Acute hepatic porphyria \\
\hline Glibenclamide & Amglidia & Neonatal Diabetes \\
\hline Glycerol Phenylbutyrate & Ravicti & Urea Cycle Disorder \\
\hline Idebenone & Raxone & Leber'S Hereditary Optic Neuropathy \\
\hline Inotersen & Tegsedi & Transthyretin Amyloidosis \\
\hline Inotuzumab ozogamicin & Besponsa & Acute lymphoblastic leukemia \\
\hline Isavuconazole & Cresemba & Invasive Aspergillosis and Mucormycosis \\
\hline Ivacaftor \& Lumacaftor & Orkambi & Cystic Fibrosis \\
\hline Ivacaftor \& Tezacaftor & Symdeko & Cystic Fibrosis \\
\hline Ixazomib & Ninlaro & Multiple Myeloma \\
\hline Lanadelumab & Takhzyro & Hereditary Angioedema \\
\hline larotrectinib & Vitrakvi & cancers with NTRK fusion \\
\hline Letermovir & Prevymis & Cmv Infection \\
\hline Limbal Stems Cells, Autologous & Holoclar & Limbal Stem Cell Deficiency \\
\hline Lutetium (177Lu) Oxodotreotide & Lutathera & Neuroendocrine Tumors (Gastroenteropancreatic) \\
\hline Mercaptamine & Cystadrops & Cystinosis \\
\hline Metreleptin & Myalepta & Lipodystrophy \\
\hline Mexiletine & Namuscla & Myotonia \\
\hline Midostaurin & Rydapt & AML \\
\hline Midostaurin & Rydapt & ASM (aggressive systemic mastocytosis) \\
\hline Migalastat & Galafold & Fabry Disease \\
\hline Nintedanib & Ofev & Idiopathic Pulmonary Fibrosis \\
\hline
\end{tabular}

Page 10/16 


\begin{tabular}{|c|c|c|}
\hline Niraparib & Zejula & ovarian cancer \\
\hline Nusinersen & Spinraza & Spinal Muscular Atrophy \\
\hline Obeticholic Acid & Ocaliva & Primary Biliary Cirrhosis \\
\hline Other Antineoplastic Agents & Zalmoxis & Haematological Malignancy (Haploidentical Hsct) \\
\hline Panobinostat & Farydak & Multiple Myeloma \\
\hline Parathyroid Hormone & Natpara & Hypoparathyroidism \\
\hline Patisiran & Onpattro & polyneuropa \\
\hline Pegvaliase & Palynziq & Phenylketonuria \\
\hline Pitolisant & Wakix & Narcolepsy \\
\hline Polatuzumab Vedotin & Polivy & Diffuse Large B-Cell Lymphoma \\
\hline Sebelipase Alfa & Kanuma & Lysosomal Acid Lipase Deficiency \\
\hline Tagraxofusp & Elzonris & Lymphoma \\
\hline Tasimelteon & Hetlioz & Sleep Disorders \\
\hline Telotristat & Xermelo & Diarrhea (Carcinoid Syndrome) \\
\hline Tisagenlecleucel & Kymriah & Large B-Cell Lymphoma, B-Cell All \\
\hline Transduced Cd34+ Cell & Strimvelis & Scid (Adenosine Deaminase Deficiency) \\
\hline Velmanase Alfa & Lamzede & Alpha Mannosidosis \\
\hline Vestronidase Alfa & Mepsevii & Mucopolysaccharidosis Vii \\
\hline Volanesorsen & Waylivra & Familial Chylomicronemia Syndrome (Fcs) \\
\hline Voretigene Neparvovec & Luxturna & Leber'S Congenital Amaurosis (Rpe65) \\
\hline
\end{tabular}

\section{Figures}

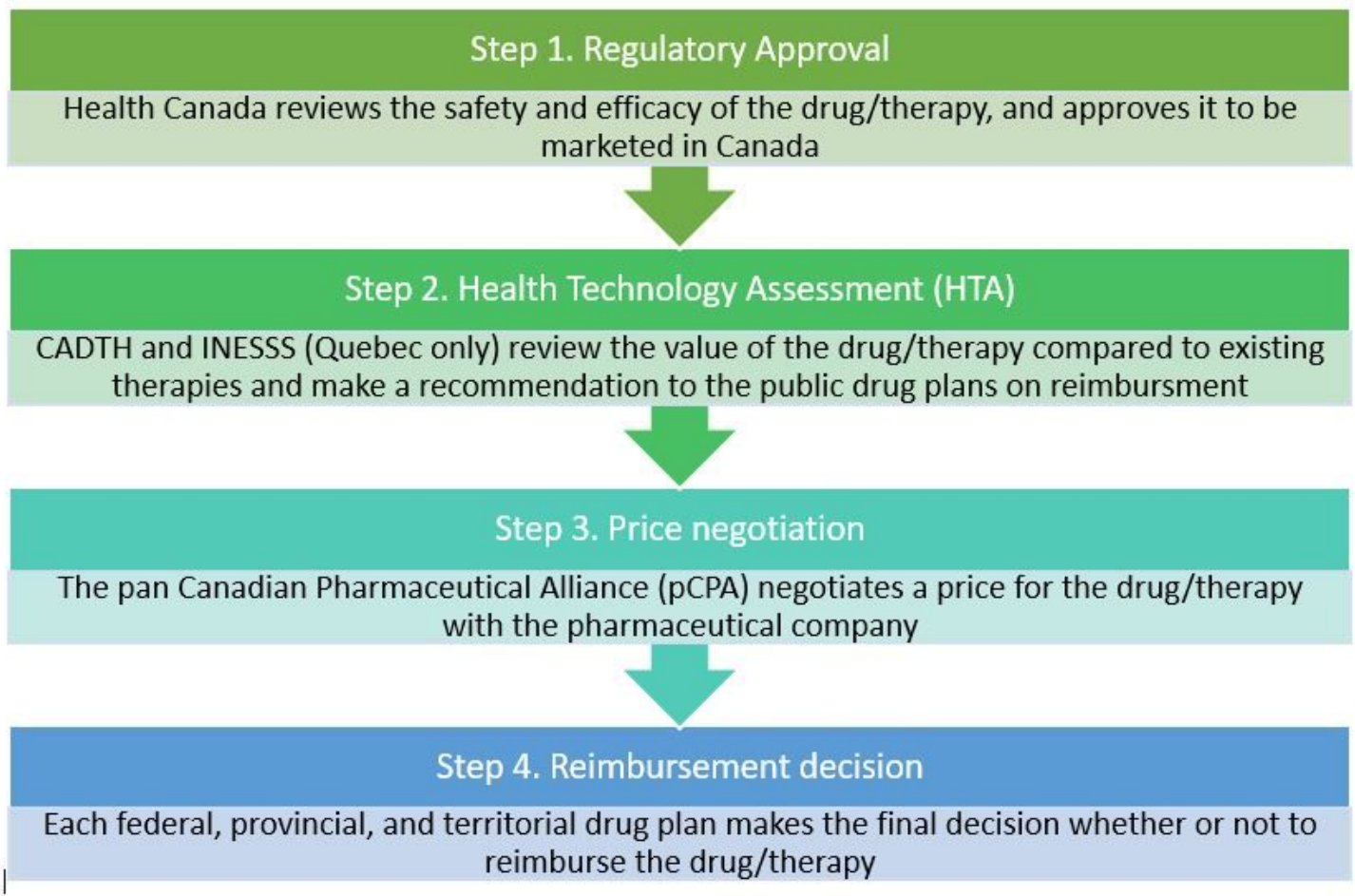

Figure 1 
Drug Reimbursement Process in Canada Abbreviations: CADTH, Canadian Agency for Drugs and Technologies in Health; INESSS, Institut national d'excellence en santé et services sociaux

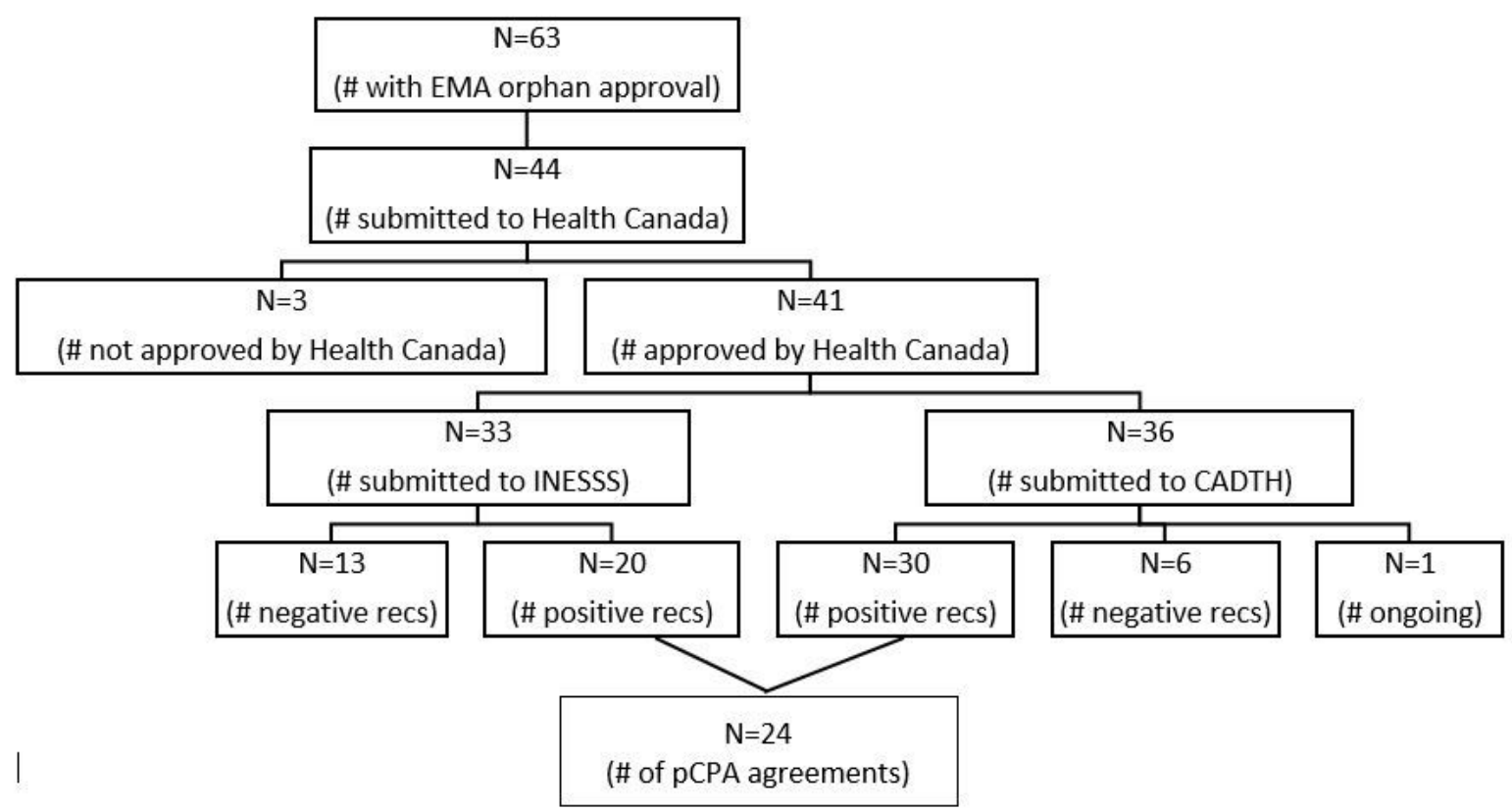

Figure 2

Flow diagram of drugs for rare diseases in the access pathway in Canada Abbreviations: CADTH, Canadian Agency for Drugs and Technologies in Health; INESSS, Institut National d'Excellence en Santé et Services Sociaux; recs, recommendations

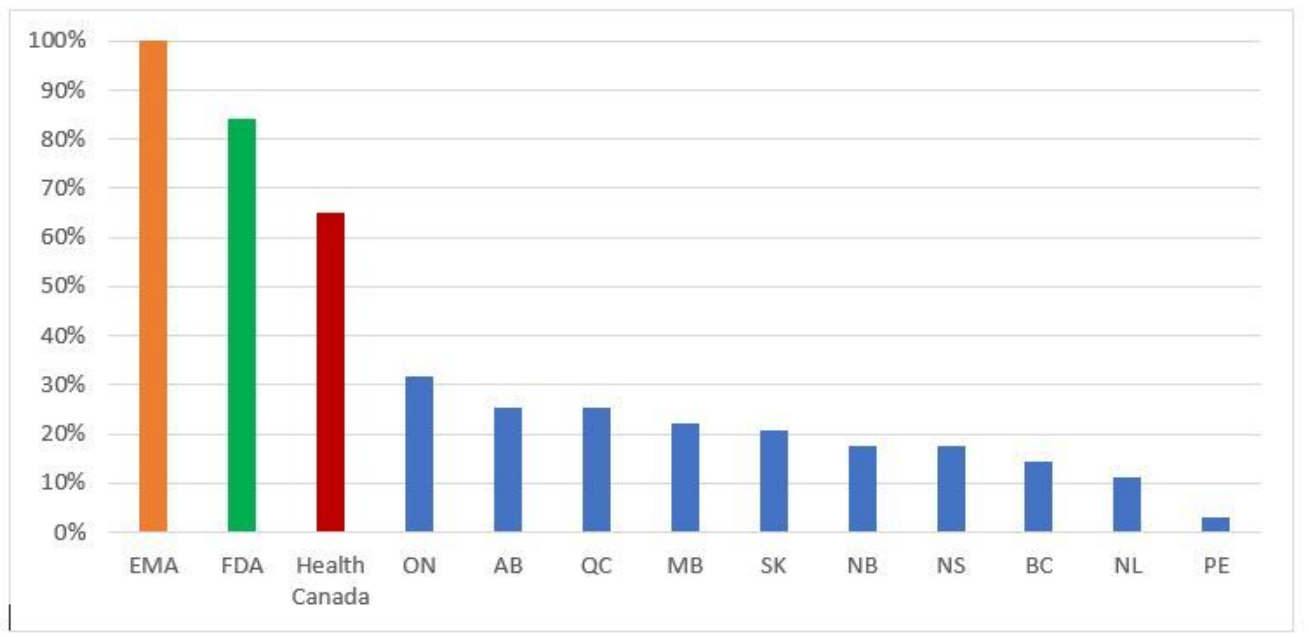

Figure 3

Percentage of drugs for rare diseases publicly funded by province compared to regulators Abbreviations: AB, Alberta; BC, British Columbia; MB, Manitoba; NB, New Brunswick; NL, Newfoundland; NS, Nova Scotia; ON, Ontario; PE, Prince Edward Island; QC, Quebec; SK, Saskatchewan 


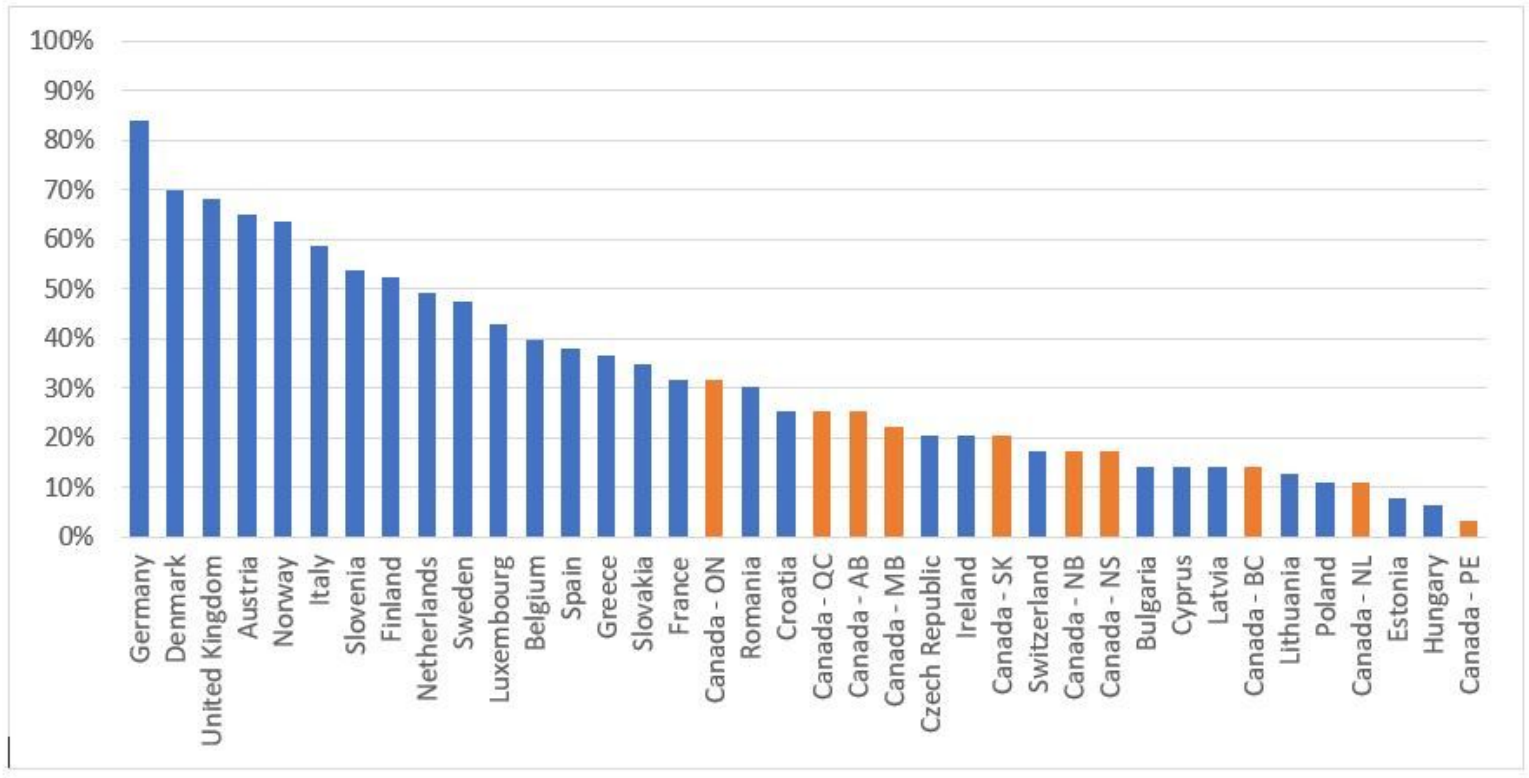

Figure 4

Percentage of therapies for rare disease that are publicly reimbursed Abbreviations: AB, Alberta; BC, British Columbia; MB, Manitoba; NB, New Brunswick; NL, Newfoundland; NS, Nova Scotia; ON, Ontario; PE, Prince Edward Island; QC, Quebec; SK, Saskatchewan

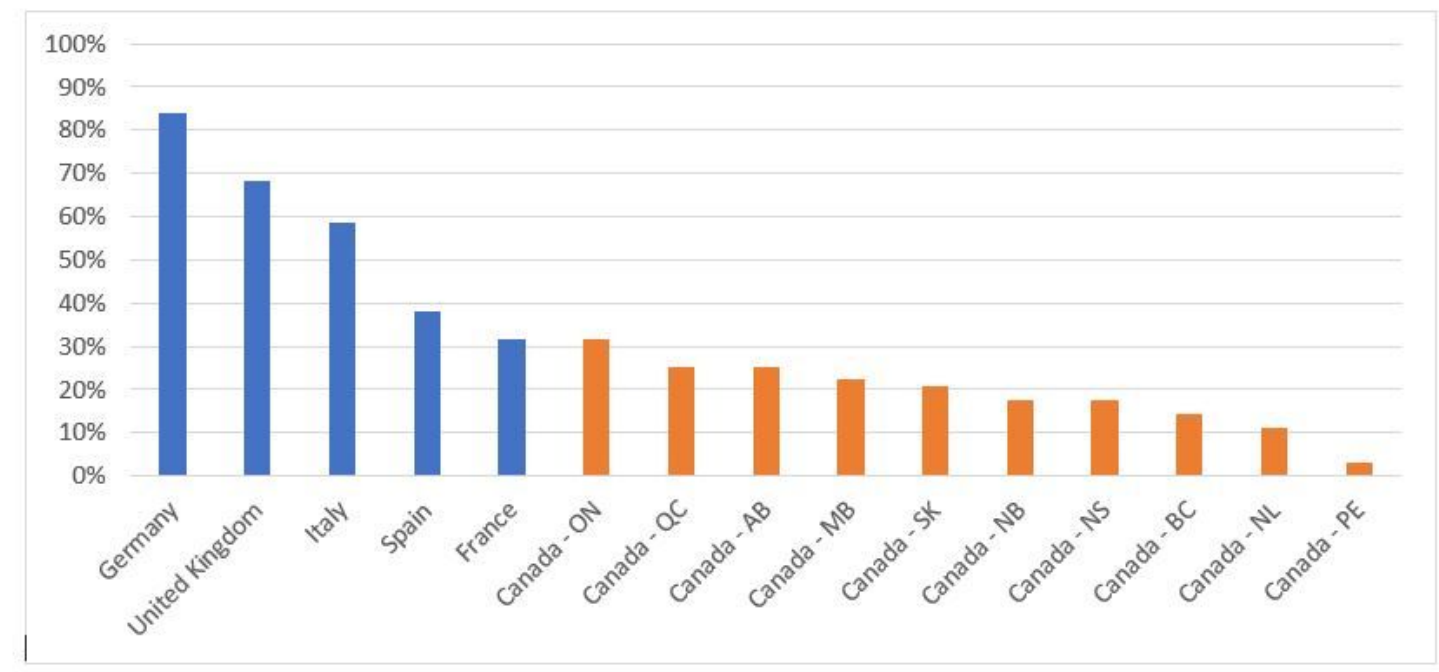

\section{Figure 5}

Percentage of drugs for rare diseases funded by province compared to the EU5 Abbreviations: AB, Alberta; BC, British Columbia; MB, Manitoba; NB, New Brunswick; NL, Newfoundland; NS, Nova Scotia; ON, Ontario; PE, Prince Edward Island; QC, Quebec; SK, Saskatchewan 


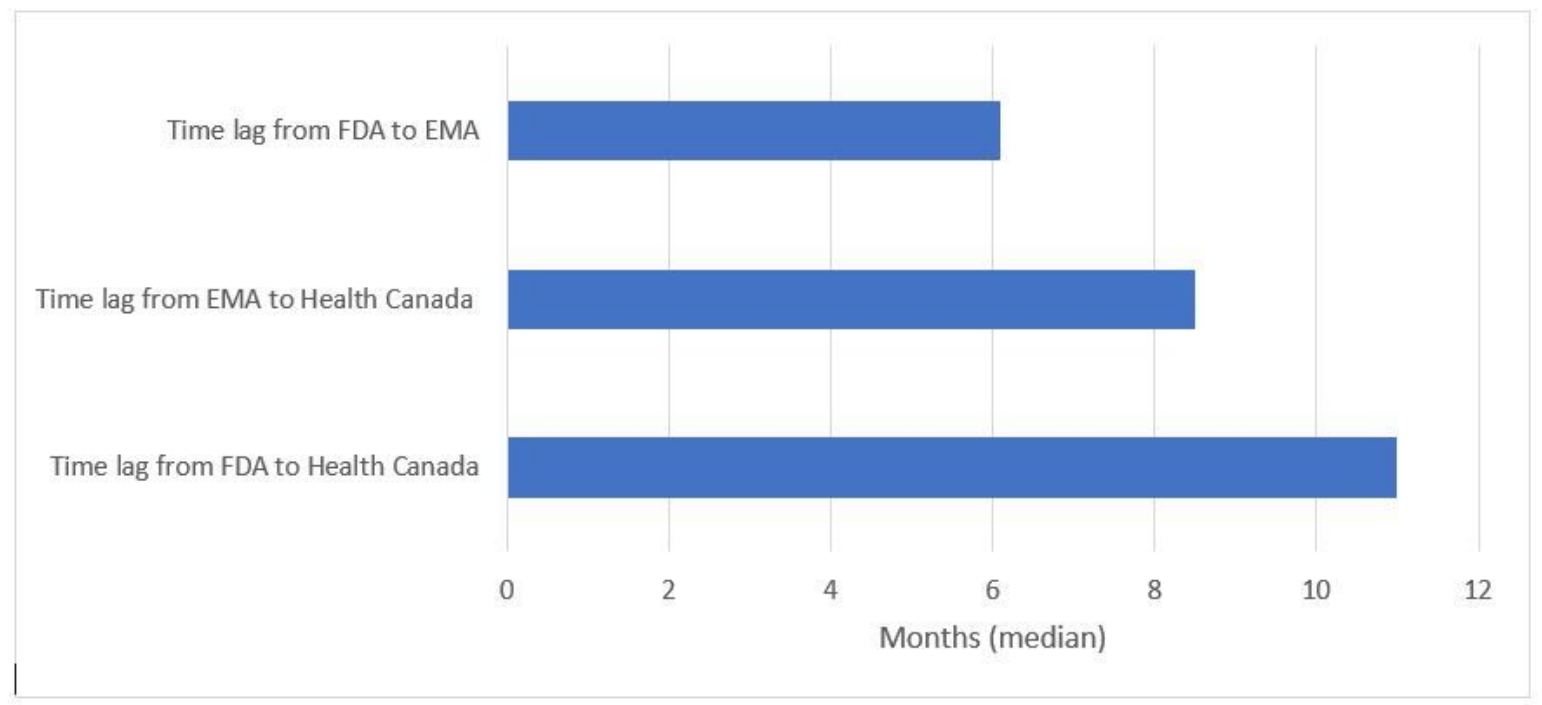

\section{Figure 6}

Comparison of regulatory approval timelines between EMA, FDA, and Health Canada

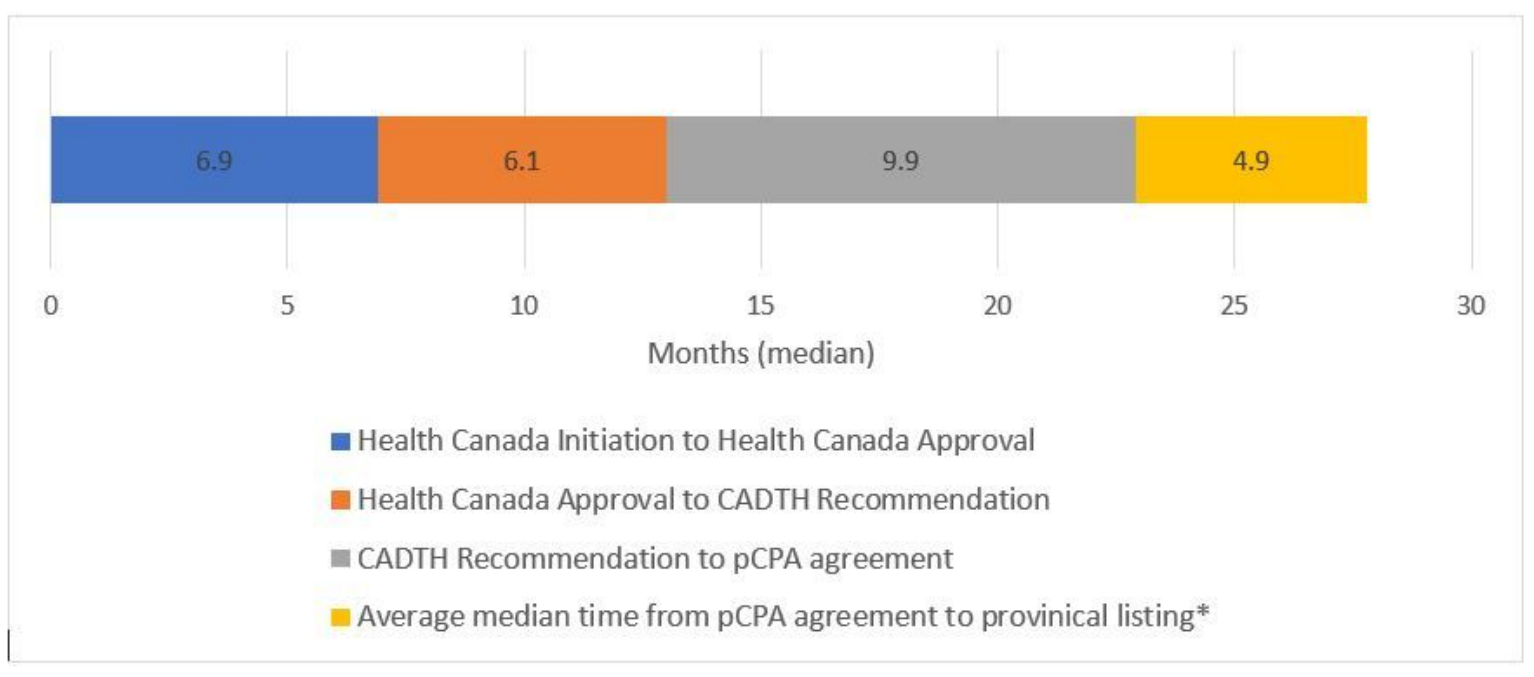

\section{Figure 7}

Median duration between steps in the Canadian drug access pathway *PEl was excluded from the average provincial median timeline calculation because the timeline for PEI was only based on 2 drugs being reimbursed 


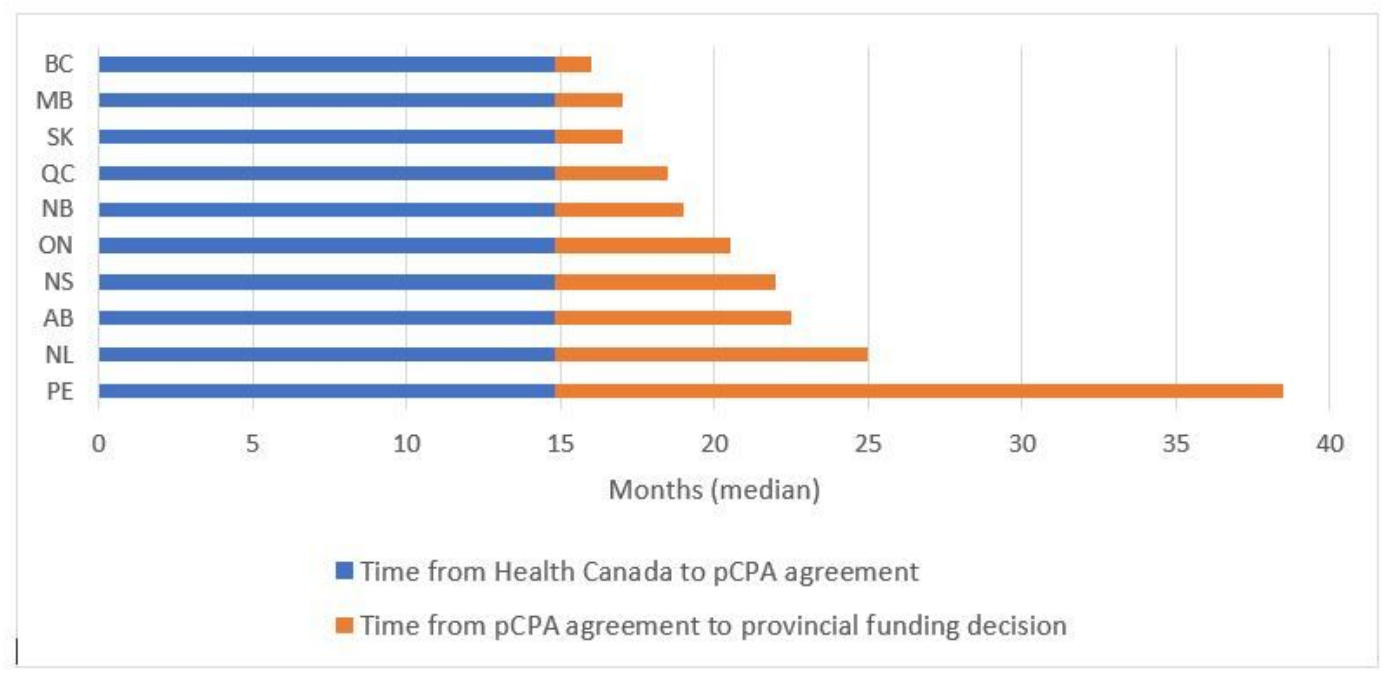

Figure 8

Mean time (months) between Health Canada approval and public reimbursement by province Abbreviations: AB, Alberta; BC, British Columbia; MB, Manitoba; NB, New Brunswick; NL, Newfoundland; NS, Nova Scotia; ON, Ontario; PE, Prince Edward Island; QC, Quebec; SK, Saskatchewan

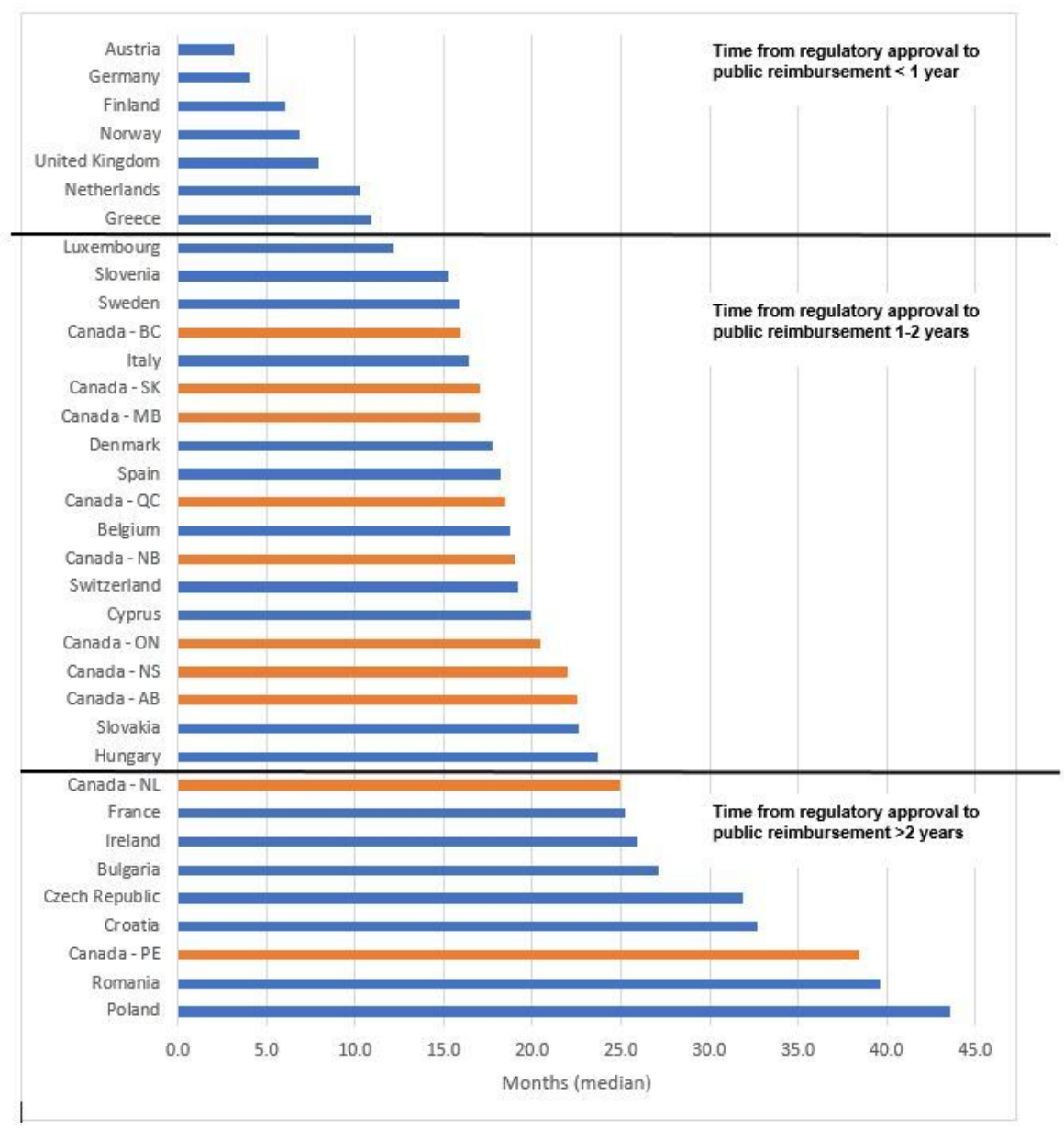

Figure 9

Timeline from regulatory approval (either EMA or Health Canada) and reimbursement Abbreviations: AB, Alberta; BC, British Columbia; MB, Manitoba; NB, New Brunswick; NL, Newfoundland; NS, Nova Scotia; ON, Ontario; PE, Prince Edward Island; QC, Quebec; SK, Saskatchewan 


\section{Supplementary Files}

This is a list of supplementary files associated with this preprint. Click to download.

- Table3.docx 